\title{
Influence De La Géomorphologie Sur La Distribution Spatiale Des Peuplements De Boscia Senegalensis (Pers.) Lam. Ex Poir. Dans La Commune Rurale De Simiri (Ouest Niger)
}

\author{
Rabiou Habou \\ Université de Diffa, Département de production végétales, \\ UMR Erémologie et lutte contre la désertification, Diffa, Niger \\ Diouf Abdoulaye \\ Université Dan Dicko Dankoulodo de Maradi, Niger \\ Inoussa Maman Maârouhi \\ Bakasso Yakoubou \\ Saadou Mahamane \\ Université Abdou Moumouni de Niamey, \\ Laboratoire Garba Mounkaila, Niger \\ Mamoudou Moussa Boubacar \\ Idi Saidou Sani \\ Laouali Abdou \\ Mahamane Ali \\ Université de Diffa, Diffa, Niger
}

Doi: 10.19044/esj.2017.v13n30p230 URL:http://dx.doi.org/10.19044/esj.2017.v13n30p230

\begin{abstract}
B. senegalensis is a species with broad geographic distribution in the Sahel, especially in Niger, thanks to its special anatomical structures to withstand drought, high temperatures and poor soils. It presents and a heterogeneous distribution in the following areas topographic levels characteristic of the western Niger. This paper aimed to assess the distribution of $B$. senegalensis and its relationship with woody species through the description of the spatial structure of the point process following toposequences by Ripley's method stands. The study highlighted the gregarious distribution of $B$. senegalensis and the close relationship between $B$. senegalensis and other species. Indeed, the species is in competition with other species on the plateau where water resources are scarce. But $B$. senegalensis tolerate these species on the slopes and in the shallows where water resources are relatively large.
\end{abstract}


Keywords: Geomorphology, spatial structure, Boscia senegalensis

\section{Résumé}

B. senegalensis est une espèce à très large distribution géographique en zone sahélienne, et en particulier au Niger, grâce à ses structures anatomiques particulière lui permettant de résister à la sécheresse, aux hautes températures et aux sols pauvres. Elle présente une répartition très hétérogène dans l'espace suivant les niveaux topographiques, caractéristiques de la partie ouest Nigérienne. La présente contribution vise à évaluer, par la méthode de Ripley, la distribution de B. senegalensis et de ses relations avec les autres espèces ligneuses à travers la description de la distribution spatiale par processus ponctuel de ces peuplements suivant les toposéquences. L'étude a permis de mettre en évidence la distribution grégaire de $B$. senegalensis et aussi de mettre en évidence les relations d'affinité entre $B$. senegalensis et les autres espèces. En effet, l'espèce est en compétition avec les autres espèces sur le plateau où les ressources hydriques sont rares. Mais $B$. senegalensis tolère ces espèces sur le versant et dans le bas fond où les ressources hydriques sont relativement importantes.

Mots clés : Géomorphologie, distribution spatiale, Boscia senegalensis

\section{Introduction}

En zones arides et semi-arides, particulièrement au Sahel, les écosystèmes sont caractérisés par une végétation ligneuse à faible productivité et soumise à des perturbations anthropiques et naturelles (Cheula, 2009). La réaction des espèces végétales face à ces perturbations diffère d'une espèce à une autre. Parmi les espèces végétales utiles recensées au Niger, B. senegalensis présente une très large distribution géographique en zone sahélienne (Salih, 1991), et en particulier au Niger, grâce à ses structures anatomiques lui permettant une grande résistance à la sécheresse et aux hautes températures et sa grande capacité de survie sur sols pauvres très dégradés et une répartition dans l'espace très hétérogène suivant les niveaux topographiques caractéristiques de la partie Ouest nigérienne (Mahamane et Saadou 2009).

B. senegalensis est un arbuste de 1 à $5 \mathrm{~m}$ de haut à large distribution au Niger : de la zone des savanes nord-soudaniennes de la Tapoa aux steppes sahariennes des vallées de l'Aïr et des plateaux de l'Irhazer (Mahamane et Saadou, 2009). C'est une espèce ubiquiste à dispositifs anatomiques divers lui permettant de stocker l'eau qu'elle utilise en saison sèche, ce qui lui confère une bonne adaptation au milieu aride (Mahamane et Saadou, 2009). L'espèce se développe sous forme de buisson toujours vert, à cime arrondie et dense. B. senegalensis est l'une des rares espèces qui portent des feuilles, 
fleurissent et fructifient en saison sèche, période de soudure alimentaire pour les populations locales qui utilisent les graines et les feuilles de cette espèce. Elle présente également des qualités pharmacologiques (Sakine et al., 2011), fourragères et alimentaires excellentes (Mahamane et Saadou, 2009).

Outre son rôle alimentaire en période de disette, cette espèce pourrait être considérée comme une alternative aux insecticides de synthèse coûteux et polluants (Doumma et Alzouma, 2006). Elle a été identifiée comme espèce-candidate dans le programme de lutte contre la désertification et la préservation de l'environnement dans le cadre de Programme Grande Muraille Verte (GMV, 2009). Mais, les données sur la sylviculture et l'écologie de l'espèce ne sont pas encore disponibles. Parmi les caractéristiques écologiques d'une espèce, la répartition spatiale de ses individus occupe une place particulière. Elle est le résultat des interactions de l'espèce avec son environnement biotique et abiotique (Traissec, 2003 ; Goreau, 2000). Mais c'est aussi une caractéristique importante de sa biologie. La répartition spatiale des individus peut conditionner la manière dont l'espèce utilise les ressources du milieu, comment elle est elle-même utilisée comme ressources.

Les variations topographiques à différentes échelles spatiales constituent une source importante de l'hétérogénéité spatiale et temporelle de la végétation. En effet, la topographie représente un gradient écologique complexe auquel sont associées des variations de types et de disponibilité en nutriments (Malenson et al. 2002), de la teneur et disponibilité en eau du sol (Boyce et al. 2005). Une forte hétérogénéité spatiale de la végétation est également intimement liée à l'hétérogénéité de l'environnement et de la distribution des espèces végétales. De plus, les espèces se répartissent en fonction de leur adaptation et de leurs exigences. De nombreuses études ont ainsi montré les relations entre les changements de la structure et composition de la végétation et les gradients topographiques (Choler et al. 2001). Kudo et al. (1999) démontrèrent la relation existant entre la distribution des types fonctionnels de plantes et l'aridité du milieu associée à la topographie.

La présente contribution vise à évaluer la distribution de $B$. senegalensis et de ses relations avec les espèces ligneuses à travers la description de la distribution spatiale des peuplements. Les questions de recherche considérées étaient les suivantes : (i) la distribution spatiale de $B$. senegalensis est-elle uniforme, indépendamment de la toposéquence ? (ii) $B$. senegalensis est-elle en compétition, en tolérance, ou en facilitation avec les autres espèces du milieu ? (iii) la distribution spatiale peut-elle favoriser la survie en mélange des espèces moins compétitives? 


\section{Matériel et méthodes}

\section{Site d'étude}

La zone d'étude est la Commune rurale de Simiri située dans le Département de Ouallam (région de Tillabéry), au Nord-Ouest du Niger entre $14^{\circ}$ et $15^{\circ}$ de latitude Nord et, $2^{\circ}$ et $3^{\circ}$ de longitude Est, couvrant une superficie de $2233 \mathrm{~km}^{2}$ (Figure 1). Les principales activités de la population sont l'agriculture pluviale et l'élevage extensif (Maiga, 2004).

La Commune rurale de Simiri se caractérise par des sols pauvres en matière organique (MO), en calcium et phosphore assimilables, et en capacité d'échange cationique (CEC) (Ambouta et Dan Lamso 1996). La structure géologique est composée de deux formations principales : les grès argileux du Continental terminal (Ct) et les sables quaternaires (Boureima, 2004). Le climat est de type tropical sec caractérisé par une courte saison de pluie ( 3 ou 4 mois) et une longue saison sèche ( 8 ou 9 mois). Les précipitations annuelles variant de $560 \mathrm{~mm}$ à $160 \mathrm{~mm}$ avec une moyenne de 378,96 \pm 85,2 $\mathrm{mm}$ par an, calculée sur les 30 dernières années (Maiga 2004). Les températures sont élevées toute l'année avec des maxima atteignant $45^{\circ} \mathrm{C}$ (avril-mai) contre $24^{\circ}$ à $26^{\circ} \mathrm{C}$ pour les minima (décembre-février considérés comme les mois les plus frais de l'année).

La végétation de la Commune rurale de Simiri est une steppe arbustive à arborée de type sahélo-saharienne (LUCOP, 2008). Les espèces arbustives sont reparties en fonction de la nature géomorphologique du substrat. Ainsi, les Combretaceae dominent sur les plateaux et les glacis tandis que Piliostigma reticulatum et Sclerocarya birrea se retrouvent au niveau des bas-fonds. La végétation en termes de densité est concentrée au Nord de la Commune, et est composée de Cenchrus biflorus et Andropogon gayanus (Maiga, 2004). 


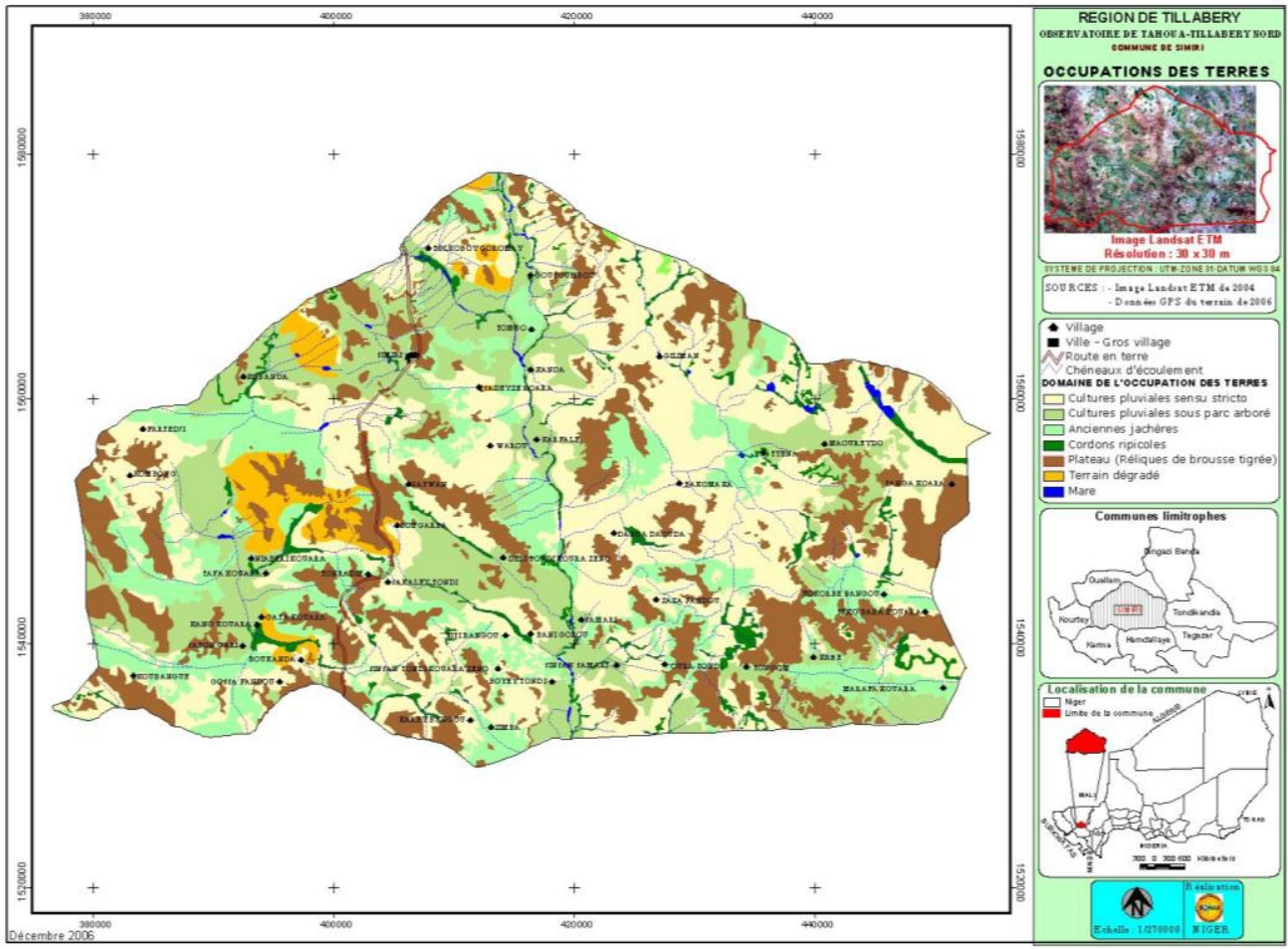

Figure 1 : Localisation de la Commune de Simiri (Source : Roselt, 2006)

\section{Collecte des données}

Trois parcelles de 1 ha $(100 \times 100 \mathrm{~m})$ chacune, ont été délimitées. Pour une meilleure collecte des données sur les individus ligneux, chaque parcelle est subdivisée en 100 quadrats de 0,01 ha chacun $(10 \mathrm{~m}$ x $10 \mathrm{~m})$, matérialisés par des piquets de fer. En effet, pour caractériser et analyser la distribution spatiale des peuplements de B. senegalensis et ses relations avec les espèces ligneuses, un transect suivant la toposéquence a été défini suivant lequel 3 parcelles équidistantes ont été délimitées, une sur le plateau, une dans le versant et une troisième dans le bas-fond.

L'analyse de la répartition spatiale des espèces ligneuses à travers l'approche par processus ponctuel (Ripley, 1977) a requis une cartographie complète de tous les individus présents, toutes espèces ligneuses confondues, dans la parcelle de relevé. Il s'est agi en fait de relever les coordonnées géographiques de chaque individu de toutes espèces confondues présentes dans le placeau. Seuls les individus ligneux possédant un diamètre supérieur ou égal à $2 \mathrm{~cm}$ ont été mesurés et cartographiés. Le choix de ce diamètre seuil s'explique par l'intervention des individus ayant ce diamètre dans la dynamique de la végétation. Les jeunes individus de diamètre inférieur à 2 cm souffrent des mortalités donc ne parviennent pas à passer la saison sèche. Ainsi, pour chaque individu, sa hauteur et les coordonnées polaires $(r, \theta)$ de 
sa position dans la parcelle sont mesurées, à l'aide d'un théodolite optique de chantier (Metland, MTXO) et d'un mètre ruban.

\section{La fonction $K$ de Ripley et les fonctions associées}

Dans le cadre de cette étude, la méthode de Ripley (1977) basée sur la théorie des processus ponctuels a été utilisée (Dixon, 2002 in Diouf 2006). Pour la caractérisation de la distribution spatiale nous avons utilisé, le Logiciel ADS in ADE4 package (http://pbil.univlyon1.fr/ADE-4/ADE4.html) ; Thioulouse et al., 1997), 1000 simulations ont été réalisées pour un risque de $1 \%$ avec une précision de $10 \%$.

La méthode la plus importante et la plus utilisée de l'analyse de processus ponctuel fut proposée par Ripley (1977) à travers la fonction $\mathrm{K}(\mathrm{r})$. Mais pour un semis de points réel, la fonction $\mathrm{K}(\mathrm{r})$ doit être estimée : l'espérance du nombre de voisins à distance $r$ d'un point quelconque est approché par sa moyenne sur l'ensemble des individus du semis. Une transformation linéaire L(r) de la fonction de Ripley a été proposée par Besag (1977) pour stabiliser sa variance (Diggle, 1983). Les fonctions K(r) et L(r) peuvent également être utilisées dans le cas où plusieurs espèces sont distinguées dans une même parcelle d'observation. En effet, ces fonctions sont utilisées pour analyser les relations spatiales qui existent entre Boscia senegalensis et les autres espèces. Pour analyser une telle répartition spatiale, on utilise la fonction $K_{i j}(r)$ qui est l'équivalent de la fonction $K(r)$ et sa fonction dérivée intertype $\mathrm{L}_{\mathrm{ij}}(\mathrm{r})$, plus facile à interpréter. Cette dernière fonction intertype a été utilisé pour l'analyse des relations spatiales qui lient $B$. senegalensis et les autres espèces les plus représentées dans les parcelles d'études. Une limite de distance de $50 \mathrm{~m}$ a été fixée correspondant à la moitié de la parcelle d'observation et des intervalles de $1 \mathrm{~m}$ pour tenir compte de l'imprécision sur les positions des individus dans la parcelle d'observation. Une approche de Monte Carlo est utilisée pour générer les intervalles de confiances et comparer la fonction L(r) d'un processus ponctuel. Les valeurs de $\mathrm{L}(\mathrm{r})$ sont inférieures à l'intervalle pour une distribution régulière et supérieur pour une structure agrégée.

\section{Resultats}

\section{Caractéristiques floristiques et dendrométriques}

Dans l'ensemble des relevés effectués dans la zone d'étude, il a été recensé 380 individus appartenant à 10 espèces ligneuses regroupées dans 9 genres et 7 familles. Les familles les mieux représentées sont les Capparaceae (20\%), les Combretaceae (20\%) et les Mimosaceae (20\%). La densité moyenne de la végétation observée au sommet du plateau est de 70 individus par ha dont 38 individus de B. senegalensis soit 54,2\%. Cette densité est plus élevée au niveau de versant avec 206 individus par ha dont 
56 individus par ha de B. senegalensis, soit $27,18 \%$. Au niveau de bas-fond la densité est de 104 individus par ha dont 29 individus du B. senegalensis par ha soit $27,88 \%$. Au niveau de versant et du bas-fond l'espèce prédominante est Guiera senegalensis avec respectivement 116 individus par ha soit $56,31 \%$ et 53 individus par ha soit 50,96\% (Tableau 1).

Tableau 1. Caractéristiques floristiques et dendrométriques

\begin{tabular}{|c|c|c|c|c|c|c|c|}
\hline \multirow[b]{2}{*}{ Espèces } & \multirow[b]{2}{*}{ Famille } & \multicolumn{2}{|c|}{ Plateau } & \multicolumn{2}{|c|}{ Versant } & \multicolumn{2}{|c|}{ Bas-fond } \\
\hline & & $\begin{array}{c}\text { Densité } \\
\text { (arbre/ } \\
\text { ha) }\end{array}$ & $\begin{array}{l}\text { Haute } \\
\text { ur (m) }\end{array}$ & $\begin{array}{l}\text { Densit } \\
\text { é } \\
\text { (arbre/ } \\
\text { ha) }\end{array}$ & $\begin{array}{l}\text { Haute } \\
\text { ur }(\mathrm{m})\end{array}$ & $\begin{array}{c}\text { Densité } \\
\text { (arbre/ } \\
\text { ha) }\end{array}$ & $\begin{array}{l}\text { Haute } \\
\text { ur }(\mathrm{m})\end{array}$ \\
\hline $\begin{array}{c}\text { Boscia } \\
\text { senegalensis }\end{array}$ & $\begin{array}{c}\text { Capparidacea } \\
\mathrm{e} \\
\end{array}$ & 38 & 1,2 & 56 & 1,06 & 29 & 1,16 \\
\hline $\begin{array}{l}\text { Combretum } \\
\text { micranthum }\end{array}$ & $\begin{array}{c}\text { Combretacea } \\
\mathrm{e}\end{array}$ & 26 & 1,54 & 33 & 1,34 & 1 & 1,4 \\
\hline $\begin{array}{c}\text { Guiera } \\
\text { senegalensis }\end{array}$ & $\begin{array}{c}\text { Combretacea } \\
\mathrm{e}\end{array}$ & 6 & 1,67 & 116 & 1,36 & 53 & 1,74 \\
\hline $\begin{array}{c}\text { Boscia } \\
\text { angustifolia }\end{array}$ & Capparaceae & - & & 1 & 1,8 & - & - \\
\hline $\begin{array}{c}\text { Ziziphus } \\
\text { mauritiana }\end{array}$ & Rhamnaceae & - & & 1 & 1,5 & 3 & 2,33 \\
\hline $\begin{array}{l}\text { Faidherbia } \\
\text { albida }\end{array}$ & Mimosaceae & - & & - & & 8 & 4,83 \\
\hline $\begin{array}{c}\text { Annona } \\
\text { senegalensis }\end{array}$ & Annonaceae & - & & - & & 1 & 1,6 \\
\hline $\begin{array}{c}\text { Balanites } \\
\text { aegyptiaca }\end{array}$ & Balanitaceae & - & & - & & 1 & 10,5 \\
\hline Acacia nilotica & Mimosaceae & - & & - & & 1 & 3,1 \\
\hline $\begin{array}{l}\text { Piliostigma } \\
\text { reticulatum }\end{array}$ & $\begin{array}{c}\text { Caesalpiniace } \\
\text { ae }\end{array}$ & - & & - & & 7 & 2,37 \\
\hline
\end{tabular}

\section{Distribution spatiale d'ensemble}

Le cordonnées récoltées sur le terrain ont permis d'établir la carte de distribution des ligneux. Les observations sur les cartes mettent en évidence des zones couvertes par la végétation et des zones relativement nues. On observe également que des individus de la même espèce forment des agrégats entre eux. Mais les agrégats sont nettement plus différenciés sur le versant, alors que sur le plateau on observe une densité faible en plante (Figure 2; 3 ; 4). 


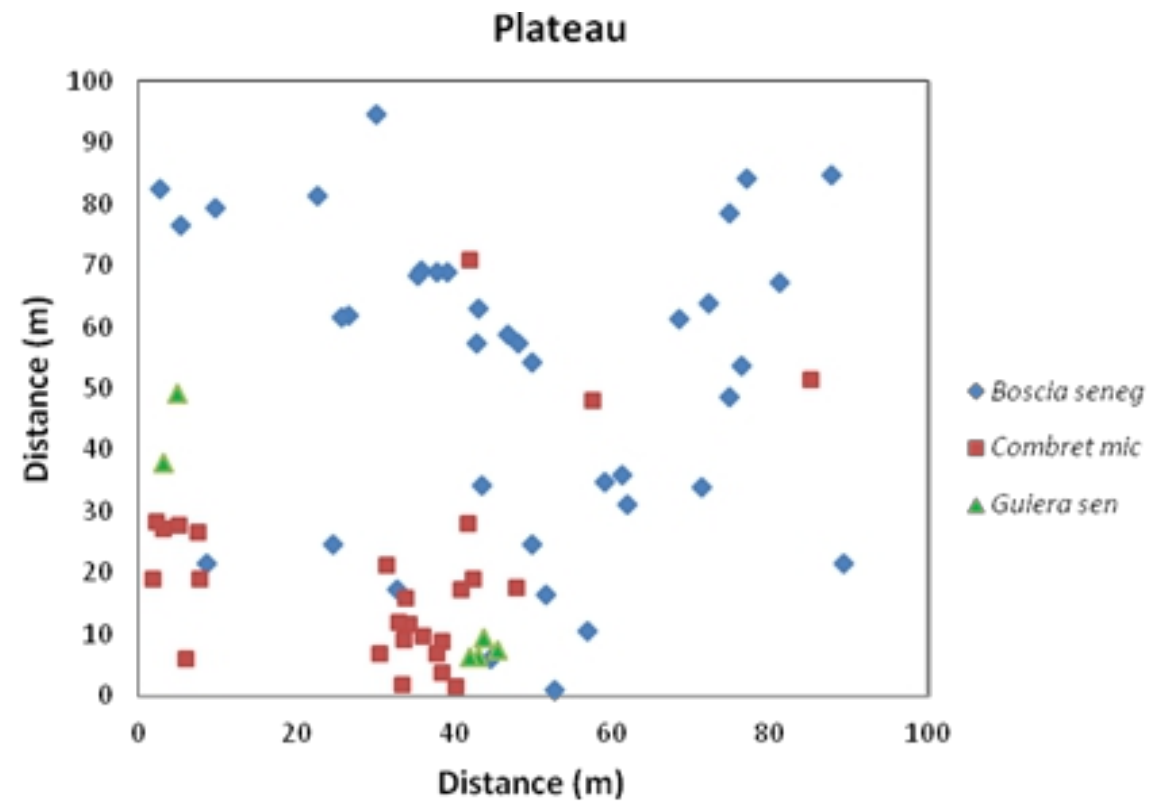

Figure 2 : Carte de distribution de tous les individus de la parcelle de plateau

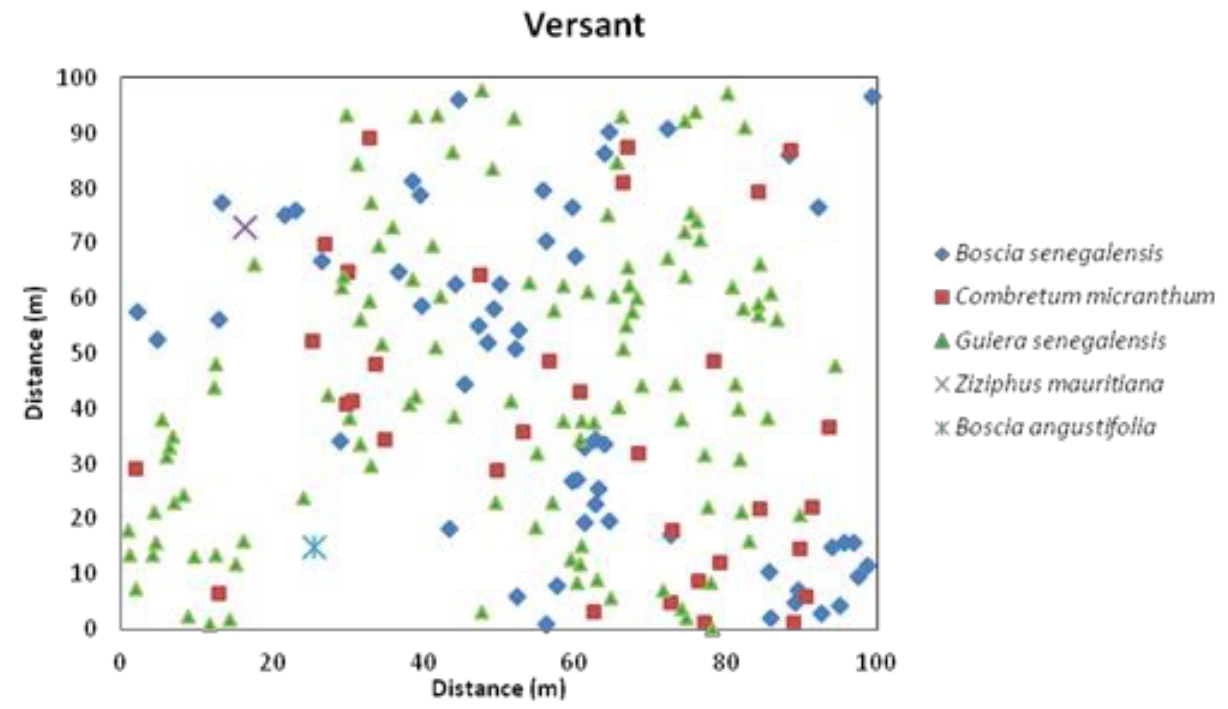

Figure 3: Carte de distribution de tous les individus de la parcelle de versant 


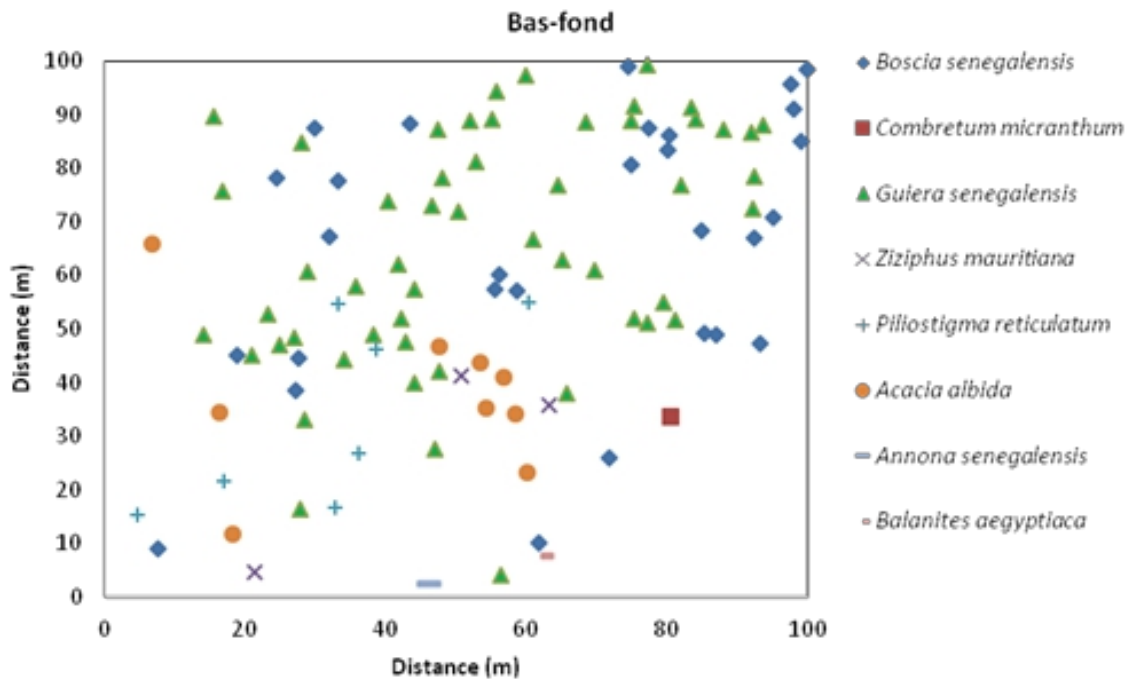

Figure 4 : Carte de distribution de tous les individus de la parcelle de Bas-fond

L'observation de ces graphiques révèle que la distribution spatiale de la végétation de la zone d'étude est hétérogène suivant la toposéquence. En effet, elles mettent en évidence le caractère épars de la végétation et le niveau topographique offrant à la végétation les conditions favorables à son développement. Une répartition plus dense observée sur le versant suivi de bas-fond, alors qu'au sommet du plateau la végétation est beaucoup moins dense. La distribution spatiale de la végétation observée sur les 3 figures peut être expliquée par l'analyse des courbes de la fonction $\mathrm{L}(\mathrm{r})$ à l'échelle des parcelles (Figure 5). La courbe de la fonction L (r) du sommet du plateau fait ressortir 2 échelles d'agrégations : les grands agrégats s'étendant sur plus de $50 \mathrm{~m}$ et à l'intérieur desquels on a des petits agrégats avec un rayon moyen de 13-16 m (Figure 5 (B)). A une plus courte (distance), la distribution des individus est aléatoire dans un rayon de $2 \mathrm{~m}$. Sur le versant, à courte distance (environ $5 \mathrm{~m}$ ) les individus se caractérisent par une distribution aléatoire. Ce qui donne lieu à des aggrégats clairsemés (figure 5 (D)) de $20 \mathrm{~m}$ de rayon moyen, et pouvant atteindre $45 \mathrm{~m}$ de diamètre. Au niveau du bas-fond, une distribution aléatoire des individus est observée à courte distance (environ $7 \mathrm{~m}$ ) et une tendance agrégative à moyenne et longue distance. Il s'agit de grands agrégats très clairsemés pouvant dépasser $50 \mathrm{~m}$ d'étendue, ce qui signifie que les individus sont plus proches les uns des autres (plus dense) qu'au niveau des versants et des bas-fonds (Figure $5(\mathrm{~F})$ ). Le non retour de la courbe dans l'intervalle de confiance est dû à l'hétérogeneité du milieu, c'està-dire que la taille des agrégats dépasse l'echelle de notre placeau d'étude.

D’une manière générale, les individus ligneux se caractérisent par une distribution aléatoire à courtes distances et une structure agrégative à 
moyennes et longues distances avec un diamètre moyen d'agrégat compris entre 46 et plus de $50 \mathrm{~m}$ selon la parcelle.

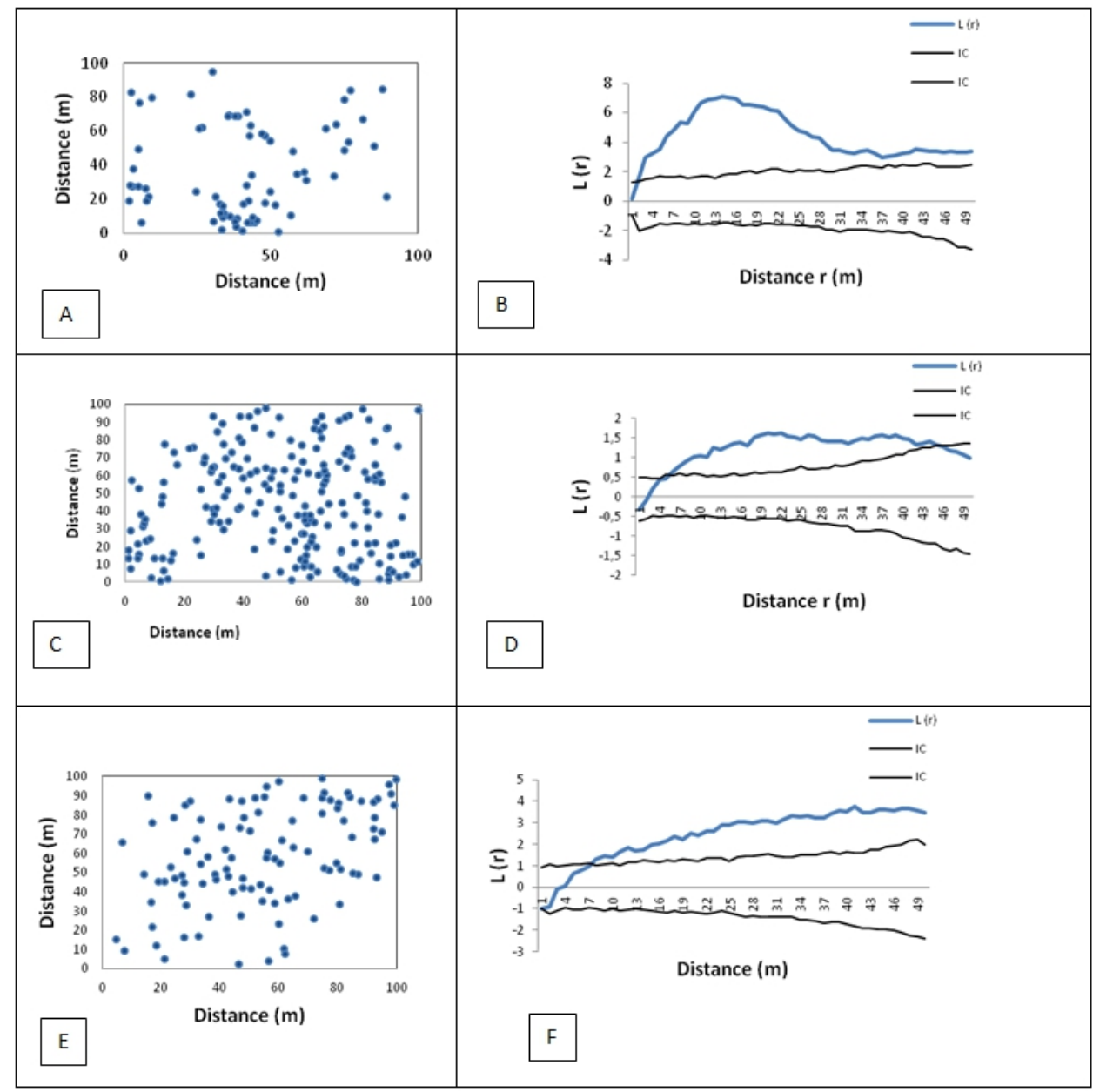

Figure 5 : Distribution spatiale des tous les individus : du plateau (A) et sa fonction $\mathrm{L}(\mathrm{r})$

(B) ; du versant (C) et sa fonction $\mathrm{L}(\mathrm{r})(\mathrm{D})$; du bas fond (E) et sa fonction $\mathrm{L}(\mathrm{r})(\mathrm{F})$.

\section{Distribution spatiale de Boscia senegalensis}

L'allure de la courbe L (r) de la Figure 6 B démontre une tendance agrégative des individus de $B$. senegalensis à courte distance $(8 \mathrm{~m})$ au sommet $\mathrm{du}$ plateau. La tendance aléatoire de la répartition spatiale de ces individus observée à très faible distance $(4 \mathrm{~m})$ revèle des agrégats clairsemés. Une agrégation clairsemée des individus s'observe également au niveau du versant, sauf que des petits agrégats de $13 \mathrm{~m}$ de rayon moyen sont imbriqués dans d'autres plus grands de $40 \mathrm{~m}$ de rayon moyen (Figure $6 \mathrm{D}$ ). La courbe $\mathrm{L}(\mathrm{r})$ présente un pic caractéristique pour une valeur de rayon correspondant approximativement au rayon des agrégats, c'est-à-dire environ $13 \mathrm{~m}$. Le pic secondaire à une distance d'environ $40 \mathrm{~m}$ correspond à l'espacement moyen 
entre les agrégats, qui constituent en quelque sorte des agrégats d'agrégats, visibles sur la carte de distribution de B. senegalensis du versant (figure $6 \mathrm{C}$ ). $\mathrm{Au}$ niveau du bas-fond, la distribution spatiale des individus de $B$. senegalensis se caractérise par des agrégats de $14 \mathrm{~m}$ de rayon moyen (Figure $6(\mathrm{~F}))$. Ces agrégats sont beaucoup plus clairsemés que dans les autres unités géomorphologiques (distribution aléatoire dans les 7 premiers mètres).

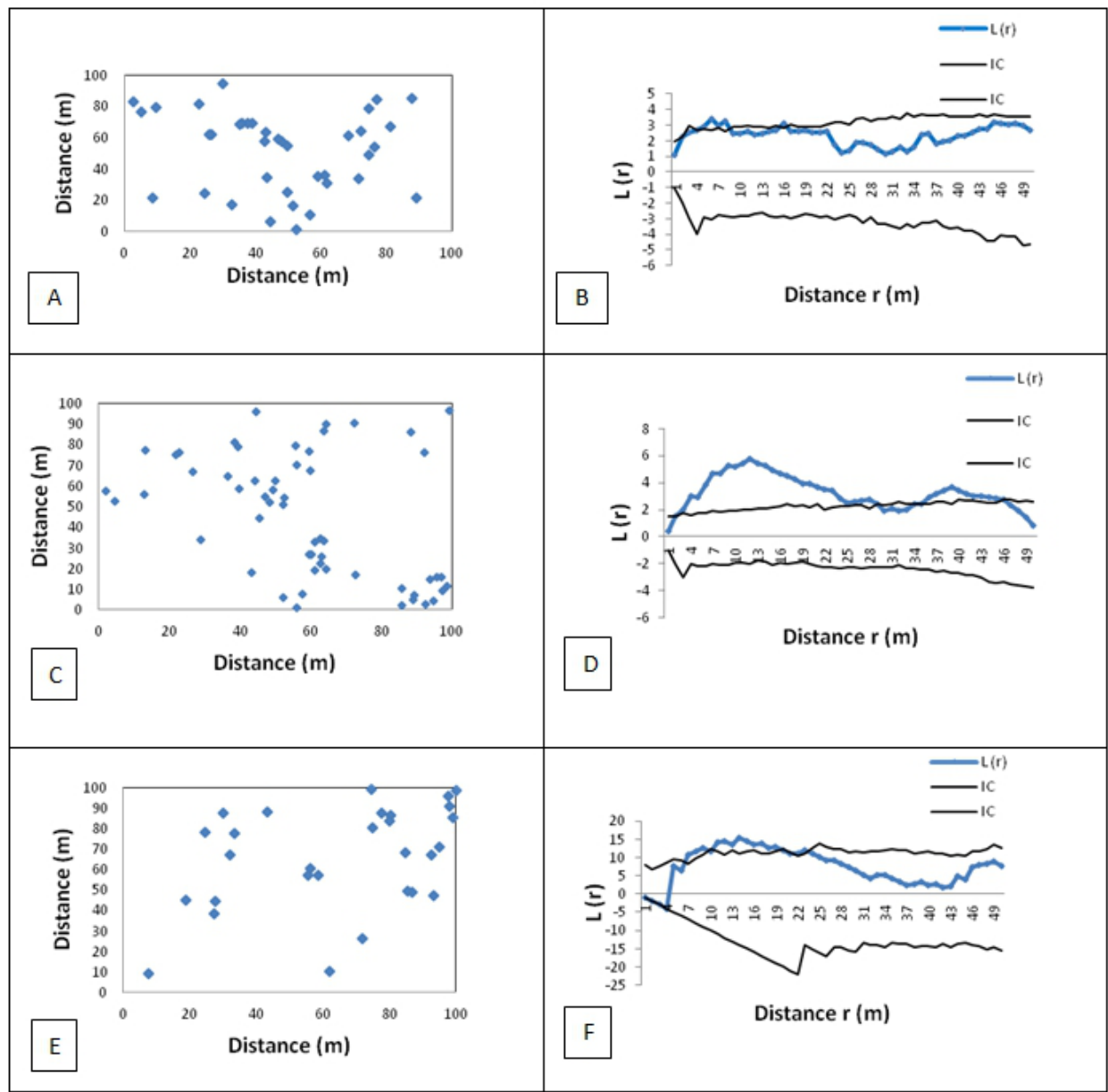

Figure 6 : Distribution spatiale des Boscia senegalensis : du plateau (A) et sa fonction L(r)

(B) ; du versant (C) et sa fonction L(r) (D) ; du bas fond (F) et sa fonction L(r) (G).

\section{Relations interspécifiques}

\section{- $\quad$ Boscia senegalensis et Guiera senegalensis}

$\mathrm{Au}$ niveau du versant, la fonction $\mathrm{L}_{12}(\mathrm{r})$ montre une distribution régulière entre les individus de Guiera senegalensis et $B$. senegalensis, cette répartition suggère une tendance répulsive, cela suppose qu'il existe une distance moyenne qui sépare chaque pied de B. senegalensis de pied de $G$. senegalensis dont la portée est de $3<\mathrm{r}<20 \mathrm{~m}$ (Figure 7 (B)). Tandis que 
dans le bas-fond l'analyse de $\mathrm{L}_{12}$ (r) montre que ces espèces ont une distribution aléatoire donc une tolérance de Boscia senegalensis vis-à-vis de Guiera senegalensis (Figure 7 (D)).

\section{- $\quad$ Boscia senegalensis et Combretum micranthum}

$\mathrm{La}$ fonction $\mathrm{L}_{12}(\mathrm{r})$ montre une forte indépendance dans la distribution de ces deux espèces au sommet du plateau (Figure 7 (F)). La carte de distribution illustre cette indépendance. Cela résulte essentiellement de l'hétérogénéité de ce plateau. En effet, le plateau renferme une grande charge en conglomérat et une rigide cuirasse, ce qui rend difficile l'installation des espèces comme $C$. micranthum qui ont des racines superficielles. Mais le dépôt d'une plage de sable a quand même permis l'installation de quelques fourrés formant une touffe de C. micranthum, visible sur la carte (Figure 7 (E)). Sur le versant, ces espèces montrent une distribution aléatoire à travers l'analyse de la fonction $\mathrm{L}_{12}(\mathrm{r})$ (Figure $7(\mathrm{H})$ ). La carte de distribution de ces espèces confirme également cette distribution aléatoire (Figure $7(\mathrm{G})$ ).

\section{- Boscia senegalensis, Piliostigma reticulatum et Faidherbia albida}

L'analyse de $\mathrm{L}_{12}$ (r) dans le bas-fond, montre que B. senegalensis et Piliostigma reticulatum ont une distribution aléatoire, et de même l'analyse de la fonction $\mathrm{L}_{12}(\mathrm{r})$ révèle que dans le même bas-fond, B. senegalensis et Faidherbia albida montre une distribution régulière d'une portée de $4<r<$ $16 \mathrm{~m}$, et avec un pic de régularité d'environ $10 \mathrm{~m}$, à travers l'analyse de la courbe de la fonction $\mathrm{L}_{12}(\mathrm{r})$. 


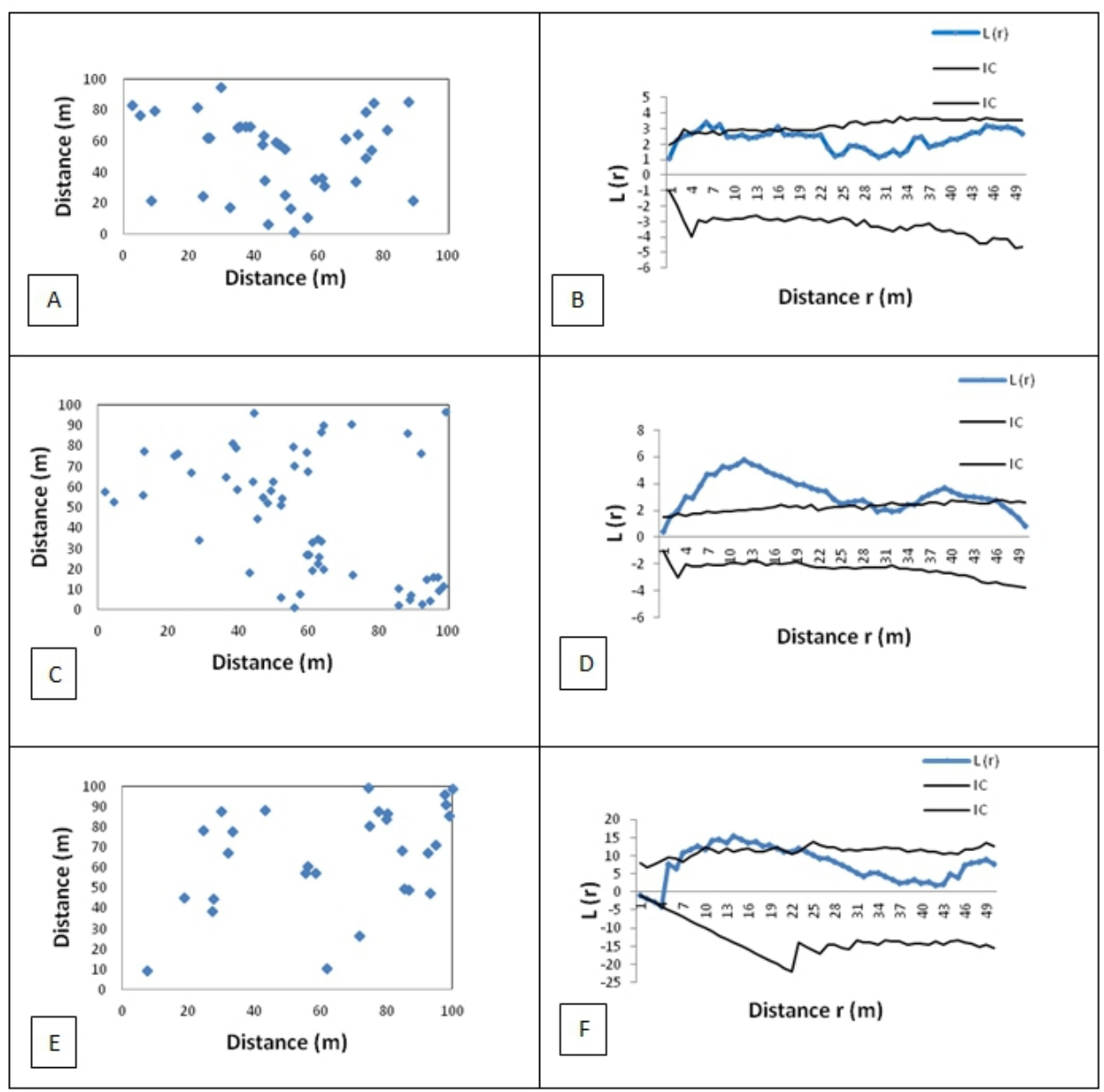

Figure 7: distribution spatiale de Boscia senegalensis et Guiera senegalensis et a fonction

L12 (r) dans le versant (A et B) et dans le bas fond (C et D); de Boscia senegalensis et Combretum micranthum et sa fonction L12 (r) sur le plateau (E et F) et sur le versant ( $\mathrm{G}$ et $\mathrm{H})$.

\section{Discussion}

\section{Attribut structuraux}

Le développement de la végétation des zones tropicales arides à semi arides est fortement lié aux rudes conditions climatiques et à la disponibilité des ressources nutritives (Deblauwe, 2010). Les espèces végétales, au nombre restreint sont ainsi contraintes de développer des stratégies adaptatives. Ainsi, en savane sahélienne, B. senegalensis est l'espèce ligneuse qui se régénère le mieux (Geider et al., 2001).

La grande plasticité de l'espèce lui confère une large amplitude écologique, d'où l'importance des superficies couvertes au Niger (Mahamane et Saadou, 2009). En effet, la présence de faisceaux surnuméraires au niveau 
des racines augmente la capacité de la plante à absorber et conserver l'eau du sol. La présence de cristaux d'oxalate de calcium de forme prismatique signalée chez les Capparaceae (Mahamane et Saâdou, 2009) explique la prépondérance de l'espèce au sommet du plateau telle que relevée dans la présente étude. B. senegalensis cohabite avec d'autres espèces, telles Guiera senegalensis et Combretum micranthum, dont la densité varie suivant le gradient topographique. Ainsi sur le versant on voit apparaitre Ziziphus mauritiana et Boscia angustifolia et dans le bas-fond on rencontre Piliostigma reticulatum, Faidherbia albida, Balanites aegyptiaca, Annona senegalensis et Acacia nilotica. Cette augmentation de la richesse spécifique s'explique par l'augmentation de l'humidité. En effet, la végétation se concentre vers les points les plus bas à bilan hydrique favorable (Karim et $a l ., 2003)$. Le sommet du plateau est très peu riche en nombre d'espèces (3 espèces/ha) et très moins dense (70 individus/ha), à cause des caractéristiques pédologiques (présence de cuirasse ferrugineuse). En effet, $B$. senegalensis possède des racines profondes et superficielles, alors que le sol au niveau de plateau est peu profond, 30 à $40 \mathrm{~cm}$ (Karim et al., 2003). Par contre $C$. micranthum et $G$. senegalensis ont des racines traçantes capables d'explorer les couches non profondes du plateau. Ces aptitudes favorisent la régénération de $C$. micranthum et $G$. senegalensis. Le versant de par ses caractéristiques pédologiques, le sol sableux du type ferrugineux tropical peu lessivé (Ambouta et Dan Lamso, 1996), favorise plus ou moins l'infiltration de l'eau et le développement de la végétation. En effet ces facteurs peuvent expliquer la densité élevée de la végétation sur le versant (Ambouta et Dan Lamso, 1996). Le bas-fond est relativement moins dense en plantes et cette situation découle du fait que la moitié de la parcelle est un champ. Le sol est alluvionnaire et constitué de dépôts de sable limoneux provenant de l'amont de la toposéquence. Avec une pente très faible, un écoulement des eaux en nappe, le ruissellement est moins agressif.

Il découle de cette situation que, $B$. senegalensis est l'une des espèces ayant un grand pouvoir d'adaptation à l'aridité, des travaux effectués par Mahamane et Saadou, 2009 ont montré que, les structures anatomiques des tiges, feuilles et racines minimisent les pertes en eau par transpiration.

\section{Distribution spatiale des espèces}

La distribution spatiale de $B$. senegalensis de manière générale est une structure agrégée mais le degré d'agrégation diffère selon l'unité géomorphologique. La forte variabilité temporelle et les fluctuations saisonnières et interannuelles des précipitations sont susceptibles de modifier des valeurs d'aridité auxquelles apparaissent des structures spatiales particulières. La distribution spatiale observée dans la présente étude montre que, pour toutes les espèces, la taille d'agrégat et les relations 
interspécifiques diffèrent d'un niveau topographique à un autre. Deblauwe (2010) a montré que les fluctuations des distributions spatiales résultent d'un équilibre naturel qui s'installe en fonction de la disponibilité des ressources. Ainsi, ces fluctuations, plutôt que de perturber les structures émergentes forcent le système à explorer différentes régions de l'espace des états créant ainsi de l'ordre là où on aurait autrement obtenu un état uniforme (végétation dense et désert) stable.

L'analyse de la distribution spatiale d'individus ligneux a permis de mettre en évidence des variations notables des répartitions selon les espèces. Toutefois les distributions agrégatives dominent généralement, entre individus d'une même espèce comme entre les individus d'espèces différentes. Cette agrégation observée à la fois à l'échelle des parcelles et au niveau des espèces est la conséquence d'une hétérogénéité environnementale induite par les plantes (phénomène de "feedback" ou rétroaction positive des plantes sur l'environnement) et d'une reproduction végétative des espèces en présence.

- Au sommet du plateau, la forte densité de B. senegalensis et sa distribution grégaire démontre la résistance de l'espèce à l'hostilité du milieu. Cette forte résistance résulte des caractéristiques xérophiles et héliophiles de l'espèce. Diouf et al. (2010) ont montré que l'espèce régénère mieux sous le couvert de $C$. micranthum dans une brousse diffuse sur le plateau de Parc W. Par contre, Akpo (1993) a montré que l'importance de la régénération de cette espèce sous couvert ligneux ne préjuge pas du devenir des plantules qui peuvent ensuite souffrir d'une mortalité importante. Pourtant, si, à l'état adulte, cette espèce est plus fréquente hors couvert, sa structure en diamètre suit une répartition bimodale, le deuxième mode (40 à $50 \mathrm{~cm}$ de circonférence à $30 \mathrm{~cm}$ du sol) représentant des spécimens sous ombrage, en majorité des individus âgés.

- Sur le versant et dans le bas-fond, la densité plus élevée (soit 3 fois celle du plateau) et la distribution grégaire de B. senegalensis s'explique par la densité élevée de la végétation. Le couvert ligneux offre en effet aux semences des conditions favorables à leur levée et à la croissance des jeunes plants, effet de l'arbre sur les facteurs microclimatiques, relèvement de la fertilité du sol (Akpo, 1993).

L'agrégation à grande échelle observée dans le cadre de cette étude résulte de l'hétérogénéité du milieu. Selon Goreaud (2000), l'hétérogénéité du milieu est liée à une forte érosion superficielle par ruissèlement et semble par contre conditionner l'installation des graines à la rencontre d'obstacles le long de la pente. Ces obstacles sont essentiellement représentés par des individus déjà en place et les jeunes se développent préférentiellement au pied des adultes où ils rencontrent un milieu favorable. 


\section{Relations intra et interspécifiques}

L'analyse de la distribution spatiale des peuplements de $B$. senegalensis autour des espèces les plus représentées a donné des résultats différents en fonction de l'espèce et de l'unité géomorphologique. En effet, il existe une relation d'affinité graduelle en fonction des espèces c'est-à-dire que $B$. senegalensis certaines espèces par rapport aux autres dans la formation des agrégats. C'est ainsi que B. senegalensis et $G$. senegalensis sont plus ou moins distantes l'une de l'autre sur le versant. Mais ces deux espèces ont une distribution aléatoire dans le bas-fond où le potentiel hydrique est important. De même $B$. senegalensis et $C$. micranthum ne forment pas des agrégats entre elles sur le plateau et ont une distribution complètement aléatoire sur le versant. On note aussi que, dans le même basfond, les pieds de B. senegalensis sont très distants des pieds de Faidherbia albida donc elles ne forment pas d'agrégats ensemble, mais $B$. senegalensis et Piliostigma reticulatum ont une distribution aléatoire. Cette situation dégage l'échelle d'affinité suivante: $B$. senegalensis- $C$. micranthum-G. senegalensis-piliostigma reticulatum-Faidherbia albida. Cette affinité entre B. senegalensis et Combretum micranthum a été signalée par plusieurs auteurs (Diouf et al., 2010 ; Jones et al., 1997 ; Gilad et al., 2004). En effet, ces résultats confortent l'hypothèse selon laquelle $C$. micranthum réduit l'évaporation directe de l'eau du sol dans les végétations périodiques de l'Afrique de l'ouest. Selon ces auteurs $C$. micranthum est donc l'espèce clé de voûte, qui de plus favorise l'installation préférentielle des espèces comme B. senegalensis et Guiera senegalensis tout autour d'elle (Diouf, 2006). L'explication à des degrés divers de compétition ou de tolérance de $B$. senegalensis avec les autres espèces réside non seulement dans la structure racinaire de ces espèces, mais aussi dans la disponibilité des ressources. En effet, $B$. senegalensis présente des racines très profondes et superficielles (Mahamane et saadou, 2009), lorsqu'elle est en présence des espèces qui ont aussi des racines pivotantes une complétion s'installe c'est le cas de $F$. albida, mais $B$. senegalensis tolère les espèces qui ont des racines traçantes qui explore la couche superficielle du sol. La disponibilité des ressources diminue le degré de compétition, c'est le cas de $B$. senegalensis et $G$. senegalensis qui sont en compétition sur le versant, ne le sont pas dans le bas-fond. Pour un peuplement forestier, la distribution spatiale détermine l'environnement local autour de chaque arbre (en particulier le nombre de voisins) et donc influence les processus naturels comme la croissance et la mortalité (Diouf et al., 2010). La distribution spatiale influence également le développement des graines et donc la régénération du peuplement. Inversement, les processus naturels ou les actions anthropiques modifient à leur tour la Distribution spatiale du peuplement qui se trouve donc dans un cycle de rétroaction (Goreaud et Pélissier, 1999; Goreaud, 2000; Pélissier et 
Goreaud, 2001). Les conditions du milieu ont une influence sur la distribution des espèces. Au fur et à mesure qu'on descend du plateau vers le bas-fond la compétition pour les ressources entre B. senegalensis et les autres espèces diminue. Ces résultats montrent aussi que la compétition est une fonction des ressources. L'analyse de la fonction intertype $L_{12}$ (r) de tous les individus montre une répulsion sur le plateau et une tolérance au niveau de versant et le bas-fond. Selon Goreaud (2000), dans un écosystème forestier, les interactions entre arbres sont bien sûr plus complexes. L'ambiance forestière, c'est-à-dire les conditions d'humidité, de température et de vent, correspond par exemple plutôt à une interaction bénéfique pour toutes les espèces. De même, les phénomènes de facilitation de la régénération par les arbres adultes (Traissec, 2003) sont des interactions positives.

\section{Conclusion}

Cette étude menée à Simiri sur Boscia senegalensis a permis de mettre en évidence la distribution spatiale $B$. senegalensis et les relations spatiales avec les autres espèces telles que Guiera senegaleneis et Combretum micranthum suivant la toposéquence. Les résultats de l'étude montrent que Boscia senegalensis est l'une des espèces capables de supporter l'hostilité des sols en zones arides.

Le choix de l'espèce dans les programmes de reboisement est justifié, compte tenu de son importance sur le plan alimentaire, pharmacologie et fourragère. L'utilisation de Boscia senegalensis comme plantation sur plateau dans le cadre de la Grande Muraille Verte (GMV) présente plusieurs avantages. En effet, l'espèce peut non seulement supporter les saisons sèches de longue durée en zone sahélienne ( 8 à 9 mois) grâce à ses structures anatomiques mais aussi, l'espèce est capable de tolérer la plupart des espèces avec lesquelles elle cohabite. Toutes fois cette cohabitation est une fonction des ressources. Le versant et le bas-fond sont des unités où le bilan hydrique est favorable, malgré le fait que $B$. senegalensis possède des dispositions anatomiques lui permettant de s'adapter à toutes les conditions des zones arides. Sur le plateau B. senegalensis cohabite avec bien d'autres espèces essentiellement des microphanérophytes comme Guiera senegalensis et Combretum micranthum dont les racines sont traçantes, alors que $B$. senegalensis possède des racines pivotantes ce qui réduit la compétition racinaire. Sur le versant et le bas-fond où les conditions sont plus favorables on trouve des mésophanérophytes comme Faidherbia albida et Piliostigma reticulatum dont la structure racinaire est pivotante, ce qui augmente la compétition avec $B$. senegalensis, malgré la disponibilité des ressources. 


\section{References:}

1. Akpo L. E. (1993). Influence du couvert ligneux sur la structure et le fonctionnement de la strate herbacée en milieu sahélien. Les déterminants écologiques, TDM, ORSTOM Paris, $174 \mathrm{p}$.

2. Ambouta K. et Dan Lamso N. (1996). Rapport d'étude des sols des terroirs de Nazey et Togom (Ouallam). Faculté d'Agronomie, Université Abdou Moumouni de Niamey, $30 \mathrm{p}$.

3. Besag J. (1977). Contribution to the discussion of Ripley's paper. Journal of the Royal Statistical Society, B39 : 193-195.

4. Boureima A. (2004). Patrimoine communautaire et conflits d'usage dans deux villages du Zarmaganda au Niger / Communuty patrimony and usage conflicts in two villages of Zarmaganda in Niger. Revue de géographie alpine, $92: 83-96$.

5. Boyce, R. L., R. Clark, and C. Dawson. (2005). Factors determining alpine species distribution on Goliath Peak, Front Range, Colorado, U.S.A. Arctic, Antarctic \& Alpine Research, 37:88-96.

6. Cheula A. (2009). Dynamique de l'occupation des sols en milieu sahélien. Espaces cultivés et couverture ligneuse dans la commune de Hombori, Mali, Mémoire Master 2, Paris 7, 44p.

7. Choler, P., R. Michalet, and. Callaway R. M. (2001). Facilitation and competition on gradients in alpine plant communities Ecological Society of America 82(12): 3295-3308.

8. Deblauwe V. (2010). Modulation des structures de végétation autoorganisées en milieu aride. Thèse de doctorat, ULB. 227p.

9. Diggle P.J. (1983). Statistical analysis of spatial point patterns. Academic Press, London: 1-148.

10. Diouf A. (2006). Etude de la distribution spatiale et des interactions biologiques au sein d'une végétation périodique du Niger par l'analyse des processus ponctuels. Mémoire DEA, ULB Bruxelles. 42p.

11. Diouf A., Nicola B., Mahamane A., Lejoly J., Saadou M., Bogaert J. (2010), Caractérisation de la distribution spatiale des individus ligneux dans une brousse tachetée au sud-ouest du Niger. Canadian journal of Forest research, 40 (4) : 827-835.

12. Gilad E., von Hardenberg J., Provenzale A., Shachak M., Meron E. (2004). Ecosystem engineers: from pattern formation to habitat creation. Phys. Rev. Lett., 93(9): 098105.

13. GMV, (2009). Critère de choix du tracé indicatif et des espèces végétales de la GMV (grande muraille verte), Modalités opérationnelles de mise en œuvre : Dogo Seck (FNRAA). Dakar. 11p.

14. Goreaud F. \& Pélissier R. (1999). On explicit formulas of edge effect correction for Ripley's K-function. Journal of Vegetation Science, 10 : 433-438. 
15. Goreaud F. (2000). Apports de l'analyse de la distribution spatiale en forêt tempérée à l'étude et la modélisation des peuplements complexes. Thèse Doctorat, Option Sciences Forestières. ENGREF, Nancy. 526p.

16. Jones C.G., Lawton J.H., Shachak M. (1997). Positive and negative effects of organisms as physical ecosystem engineers. Ecology, 78(7): 1946-1957. [ISI]

17. Kudo, G., U. Nordenhall, and U. Molau. (1999). Effects of snowmelt timing on leaf traits, leaf production, and shoot growth of alpine plants: comparisons along a snowmelt gradient in northern Sweden. Ecoscience 6:439-450.

18. Lucop, (2008): Les activités de gestion et mise en valeur des ressources naturelles dans la région de Tillabéri ; Programme NigéroAllemand de Lutte contre la Pauvreté Tillabéri et Tahoua-Nord. 7p.

19. Mahamane. A et Saadou. M, (2009). Structures anatomiques de quelques organes de Boscia senegalensis (Pers.) Lam. ex Poir. Et adaptation à la sécheresse. Sécheresse $2009 ; 20$ (2) : 237-9.

20. Maiga F., (2004). L'érosion éolienne dans le Zarmaganda (Niger) / Wind erosion in Zarmaganda In: Revue de géographie alpine. 2004, Tome $92 \mathrm{~N}^{\circ} 1$. pp. 71-82.

21. Malenson, G. P., D. R. Butler, D. M. Cairns, T. E. Welsh, and L. M. Resler. (2002). Variability in an edaphic indicator in alpine tundra. Catena 49:203-215.

22. Pélissier R. - (1995) : Relations entre l'hétérogénéité spatiale et la dynamique de renouvellement d'une forêt dense humide sempervirente (Forêt d'Uppangala - Ghäts occidentaux de l'Inde). Thèse, Lyon I. 237 p.

23. Pélissier, R. \& Goreaud, F. (2001). A practical approach to studying the spatial structure in simple cases of heterogeneous vegetation stands. Journal of Vegetation Science, 12: in press.

24. Ripley, B.D. (1977). Modelling spatial patterns. Journal of the Royal Statistical Society, B39: 172-212.

25. Salih. O, .M., Nour, A .M. \& Harper, D.B. (1991). - Chemical and Nutritional Composition of Two Famine Food Sources Used in Sudan, Mukheit (Boscia senegalensis) and Maikah (Dobera roxburghi). J. Sci. Food Agric., 57 : 367-377.

26. Sakine M. N. A., Mahmout Y., Gbenou J., Agbodjogbe W., Moudachirou M., (2011). Effet antihyperglycémiant des extraits de Boscia senegalensis (Pers.) Lam. ex Poiret et de Colocynthis vulgaris (L.) Schrad. Phamathérapie, Springer-Verlag France, 9: 268-273. DOI: 10.1007/s10298-011-0650-5. 
27. Doumma. A et Alzouma. I, (2006) : Influence de Boscia senegalensis (Pers) Lam. Ex Poir. (Capparaceae) sur les capacités de dispersion de Dinarmus basalis Rond. (Hymenoptera-Pteromalidae) dans les systèmes de stockage traditionnels de niébé. Tropicultura, 24, 4, 208212

28. Traissec S. (2003). Dynamique spatiale de Vouacapoua americana (Aublet), arbre de forêt tropicale humide a répartition agrégée. Thèse de doctorat, Université Claude Bernard, Lyon 1, p. $201+$ Annexe.

29. Geider, R. J., Delucia E. H., Falkowski P. G., Finzi A. C., Grime J. P., Grace J., Kana T. M., La Roche J., Long S. P., Osborne B. A., Platt T., Prentice I. C., Raven J. A., Schlesinger W. H., Smetacek V., Stuart V., Sathyendranath S., Thomas R. B., Vogelmann T. C., Williams P., And Woodward F. I., (2001). Primary productivity of planet earth: biological determinants and physical constraints in terrestrial and aquatic habitats. Global Change Biology 7:849-882.

30. Karim S., Bationo B. A., Bellefontaine R., Ichaou A., (2003). Reboiser au moindre coût les zones semi-arides par marcottage naturel, XIIè Congrès Forestier Mondial (Montréal), sept. 2003. 